IV. Aus der Universitäts-Augenklinik in Breslau.

\section{Ein Beitrag zu den selteneren Formen der Sehstörungen bei intracraniellen Er- krankungen.}

Von Prof. W. Uhthoff.

(Schluss aus No. 9.)

II. Ein Fall von linksseitiger homonymer Hemianopsie mit Uebergreifen auf die rechten Gesichtsfeldhälften, complicirt mit doppelseitiger Ophthalmoplegia interna und Morbus Basedowii.

Pauline H., 28 Jahre alt, Dienstmädchen aus Breslau, wurde am 26. October 1897 in die Klinik aufgenommen. Sie giebt an, seit circa acht Wochen oine Verschlechterung ihres Schvermogens und namentlich nach links heruber bemerkt zu haben. Schon seit Juli 1897 leidet sie viel an rechtsseitigen Kopf-, Gesichts- und Zahnschmerzen, die fast alle Tage während mehrerer Stunden, namentlich aber Abends, mit grosser Heftigkeit auftraten. Gelegentliche Ohnmachtsanfälle. Vor neun Jahren hat Patientin an "Gelenkrheumatismus" längere Zeit gelitten. - Vor einigen Jahrcn Gesichtsausschlag (Iuetisch?). - Sonst wird in hereditärer Hin-

Fig. 43.

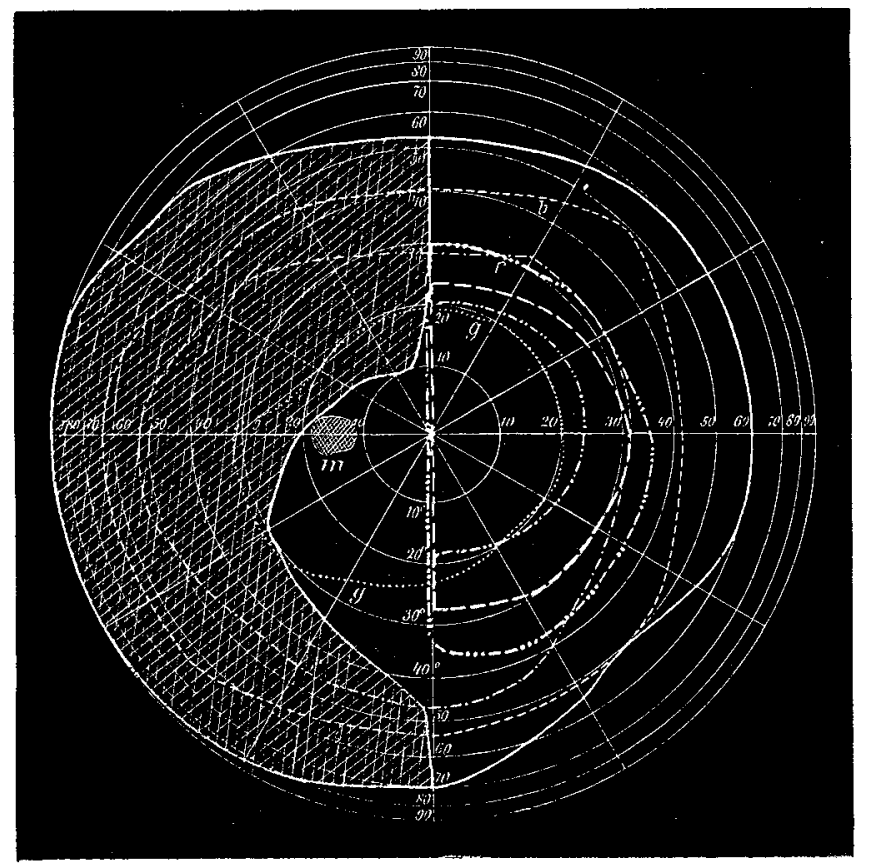

sicht nichts angegeben. Speziell Infection wird nicht zugegeben, doch lat Patientin Gelegenheit dazu gehabt.

Die objective Untersuchung ergiebt in Betreff der Augen: Rechtes Auge $\mathrm{ES}=6 / 12$, Sn 1,3 p pr $30 \mathrm{~cm}$, mit $+4,0 \mathrm{D}$, Sn 0,5 ; linkes Auge ES $=6 / 6$, Sn 0,8 p pr $30 \mathrm{~cm}$, mit $+4,0 \mathrm{D}$, Sn 0,5 .

Es besteht somit beiderseits eine ausgesprochene Parese der Accommodation. Beiderseits keine Reaction der Pupillen auf Licht, auf Convergenz auch nur sehr geringe Verengerung. Die rechte Pupille weiter als die linke (Ophthalmoplegia interna). Die Augenbewegungen sonst frei

Ophthalmoskopisch: Rechts leichte, aber deutliche atrophische $\mathrm{Ab}$ blassung' der Papille, Grenzen scharf, Retinalvenen etwas abnorm ge schlängelt. Links Befund noch im wesentlichen normal. Im Verlaufe der Beobachtung leichte atrophische Verfärbung.

Die Gesichtsfelder zeigen eine ausgesprochene linksseitige Hemianopsie, jedoch der Art, dass ein weisses Object noch etwas über die vertikale Trennungslinie hinaus erkannt wird, während links die Farbengrenzen im hemianopischen Sinne scharf mit der Mittellinie abschneiden. Rechts werden Farben auch in der rechten Gesichtsfeldhälfte überhaupt nicht mehr erkannt, ebenso greift hier der absolute Gesichtsfelddefect im oberen äusseren Abschnitt deutlich in die rechte Gesichtshälfte tuber. (S. Fig. 43 u. 44.)

Es ergiebt sich somit, dass wir es hier mit einer linksseitigen homonymen Hemianopsie zu thun haben, die durch direktes Weiterschreiten auch die äussere Gesichtsfeldhälfte des rechten Auges in Mitleidenschaft gezogen hat. Der weitere Verlauf der Beobachtung bestätigt diese Auffassung.

Ein solches Gesichtsfeldverhalten ist nur erklärlich bei einer Affection der vorderen rechten Tractuspartieen mit Uebergreifen des Krankheitsprocesses auf das Chiasma selbst.

Da die Lichtreaction der Pupillen überhaupt fehlte, konnte hier naturlich von einer Untersuchung auf hemianopische Pupillenreaction nicht die Rede sein.
Die sonstige Untersuchung der Patientin ergab folgendes an krankhaften Veränderungen: Puls sehr beschle unigt, 120-140 in der Minute gleichmässig, ziemlich kräftig, Herzstoss verstärkt, etwas nach links verschoben.

Ziemlich starker, schnellschlägiger Tremor der Hände. Patientin ist psychisch sehr leicht erregbar, Hyperhidrosis, ausgesprochene Dermographie.

Schmerzen bei Druck auf die untersten Intercostalräume, ausgesprochene Hyperalgesie daselbst, die sich auch bis nach der Wirbelsäule hin nach hinten erstreckt, in dieser Zone auch subjectiv zeitweise "reissende" Schmerzen.

Die Patellarreflexe sind gesteigert, doch bestehen keine Sensibilitätsstörungen an den Beinen.

Es besteht nun ferner eine ausgesprochene Struma. Die Scliild drüse ist in ihrer mittleren Partie hauptsächlich vergrössert und von derber Consistenz. Diese Struma besteht schon seit dem 14. Lebensjahr, hat sich aber in der letzten Zeit vergrossert.

Es handelt sich hier trotz fehlendem Exophthalmus unzweifelhaft un den Symptomcomplex der Basedow'schen Krankheit neben dem sonstigen complicirten Augenbefund. Diese Auffassung wird auch rückhaltlos von neurologischer Seite (Dr. Mann) getheilt und ebenso auf Grund einer internen Untersuchung (Dr. Weintraud).

Fig. 44.

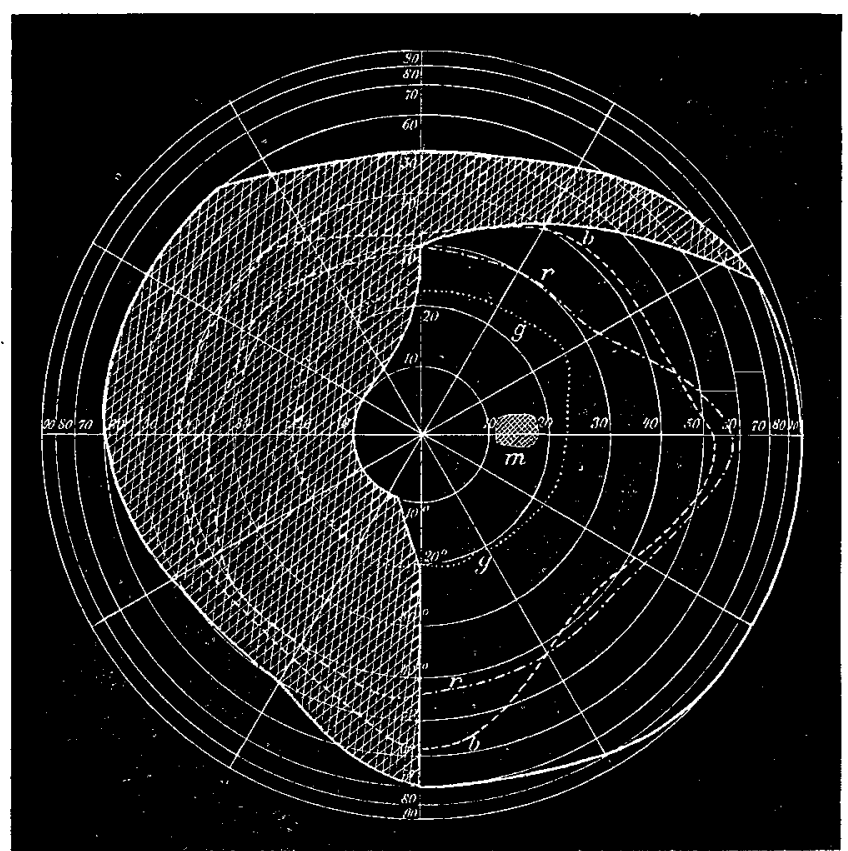

In der Inguinalgegend beiderseits indolente Ijynıhdrusenschwellung. Der Urin war normal, Menge zeitweise etwas abnorm reichlich. Sonst keine krankhaften Veränderungen des Körpers.

Während ihres klinischen Aufenthaltes machte Patientin eine ausgiebige Schmiercur durch mit gleichzeitigem innerlichen Gebrauch von Jodkalium. Später noch Bromkalium und Anwendung des constanten Stromes. Vorubergehend wurden auch Thyreoidintabletten verabreicht.

Während dieser klinischen Behandlung hielten sich die Sehschärfe und die Gesichtsfelder anfangs auf demselben Standpunkt, später ging die Gesichtsfeldbeschränkung etwas, aber deutlich zurück. (S. Fig. 45 und 46, S. 172.)

Das Allgemeinbefinden war zeitweise recht schlecht. Patientin hatte oft sehr starke rechtsseitige Kopfschmerzen, die Herzpalpitationen bestanden Wochen hindurch continuirlich, wenn auch der Intensität nach ctwas wechselnd. Starke psychische Erregungszustände, 'Tremor wechselnd, aber schon bei geringen psychischen Erregungen sehr ausgesprochen. Hyperhidrosis sehr hervortretend. Starke Abnahme des Körpergewichts (20 Pfund)

Gegen Schluss der klinischen Behandlungszeit gingen die Erscheinungen etwas zurtick, und am 23. December 1897 wurde sie als gebessert entlassen.

Das subjective Befinden ist zu dieser Zeit bedeutend gebessert, Patientin ist frei von Kopfschmerzen, der Schlaf meist gut (früher zeitweise Schlaflosigkeit). Der Tremor viel geringer, Puls durchschnittlich 110 in der Minute, Herzstoss etwa von normaler Stärke. Dermographie besteht fort. Die Hyperalgesie am Thorax völlig geschwunden, keine Druckempfindlichkeit mehr daselbst, die Steigerung der Patellarreflexe ist weniger ausgesprochen als fruher.

Auch jetzt schon, einige Wochen seit der Entlassung aus der Klinik, ist das Allgemeinbefinden der Patientin relativ gut, sie hat an Körpergewicht zugenommen, die nervősen Erscheinungen (Tremor, Unruhe, Aengstlichkeit, Schlaflosigkeit u. s. w.) haben sich erheblich gebessert, 
die rechtsseitigen Kopfschmerzen sind geschwunden, auch die Herzpalpitationen haben nachgelassen. Patientin ist in ihre fruhere Stellung zurückgekehrt und verrichtet leichtere Arbeiten. Die Sehstorung und das Gesichtsfeldverhalten sind ungefähr dieselben, wio bei der Entlassung, nur tritt auch links in der letzten Zeit eine deutliche, einfach atrophische Verfarbung der Papille $z u$ Tage, die auf dem rechten Auge ebenfalls noch zugenonimen hat.

Fig. 45.

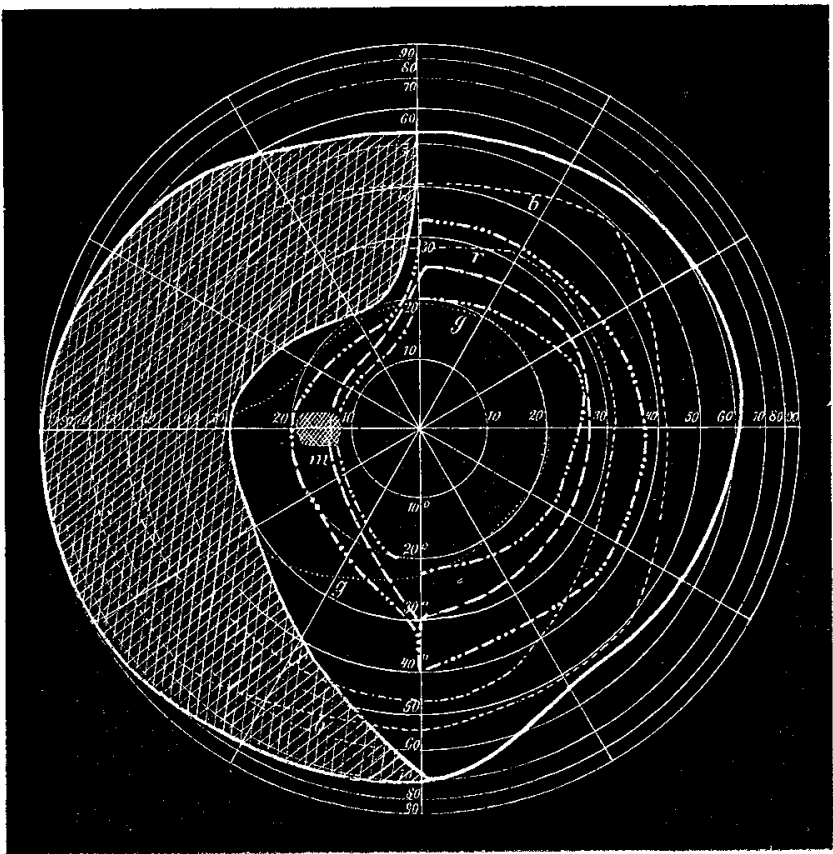

Es handelt sich hier somit um complicirte Krankheitserscheinungen bei der Patientin, die in linksseitiger Hemianopsie mit Uebergreifen auf die rechte Gesichtsfeldhälfte des rechten Auges, doppelseitiger Ophthalmeplegia interna ohne sonstige Bewegungsstörungen der Augen, Struma und sonstigen ausgesprochenen Erscheinungen des Morbus Basedowii (Herzpalpitationen, Tremor, Hyperhidrosis, psychische Erregungszustände u. s. w., aber ohne Exophthalmus) bestehen. Es fragt sich nun zunächst, ob man berechtigt ist, alle diese Erscheinungen als Componenten eines einheitlichen Krankheitsprocesses aufzufassen.

Die Sehstörung unter dieser eigenartigen Gesichtsfeldbeschränkung auf beiden Augen weist, meines Erachtens, mit Sicherheit auf das vordere Ende des rechten Tractus opticus mit Uebergreifen des Processes auf das Chiasma selbst. Sowohl die Ausbildung als auch die Rückbildung der Sehstörung spricht für diese Lokalisation, es ist nicht angängig, das Uebergreifen der Gesichtsfeldstörung auf die rechte Gesichtsfeldhälfte des rechten Auges durch einen zweiten, weiter centralwärts gelegenen Heerd in der linken Hemisphäre ¿u erklären; denn dann dürfte die rechte $\mathrm{Ge}$ sichtsfeldhälfte des linken Auges nicht intact geblieben sein. Eine solche Asymmetrie bei dem Fortschreiten des Gesichtsfeldverfalles ist nur möglich, wenn die Kreuzungsstelle (Chiasma) in Mitleidenschaft gezogen wurde. Es ist dies der dritte Fall, wo ich bisher eine zunächst einfache homonyme Hemianopsie durch direktes Fortschreiten des Processes auf die bis dahin gesunden Gesichtsfeldhälften übergreifen sah, und zwar in einer Weise, die nur eine Lokalisation im vorderen Tractusende mit Uebergreifen auf das Chiasma zuliess. In dem einen der beiden anderen Fälle handelte es sich um eine basale syphilitische Affection des einen vorderen Tractusendes, die daun auf das Chiasma überging (v. Graefe's Archiv für Ophthalmologie Bd. XXXIX, No. 3 "Ueber die bei der Syphilis des Centralnervensystems vorkommenden Augenstörungen “) unter gleichzeitiger atrophischer Verfärbung der Papillen. Der zweite Fall betraf einen Patienten mit Akromegalie („Ein Beitrag zu den Sehstörungen bei Zwergwuchs und Riesenwuchs, resp. Akromegalie" Berliner klinische Wochenschrift 1897, No. 22) mit einer solchen Gesichtsfeldanomalie und gleichzeitiger Convergenzparese, bei dem die Autopsie eine Geschwulst (Sarkom) in der Hypophysisgegend mit Betheiligung der Sella turcica ergab.

Gerade mit Rücksicht auf diesen letzten Fall könnte man ja vielleicht geneigt sein, auch bei unserer jetzigen Patientin die Sehstörung auf eine krankhafte Veränderung der Hypophysis zurückzuführen und die letztere wiederum in Zusammenhang $\mathrm{zu}$ bringen mit der Degeneration in Vergrösserung der Schilddrüse, die andererseits auch wieder die Erscheinungen der Basedow'schen Krankheit bedingt haben könnte.

Die früheren Untersuchungen ron Rogowitsch, Stieda, v. Eiselsberg, Lancereaux, Horsley, Moussu, Hofmeister sind auch in neuester Zeit noch von anderen Untersuchern (Leonhardt, Virchow's Archiv für pathologische Anatomie Bd. CIXL, S. 341 u. a.) erweitert worden, die ebenfalls nach Schilddrüsenexstirpation Vergrösserung der Hypophysis sahen. Besonders aber sei hier noch einmal auf die Schoenemann'schen Untersuchungen („Hypophysis und Thyreoidea“, Virchow's Archiv für pathologische Fir. 46.

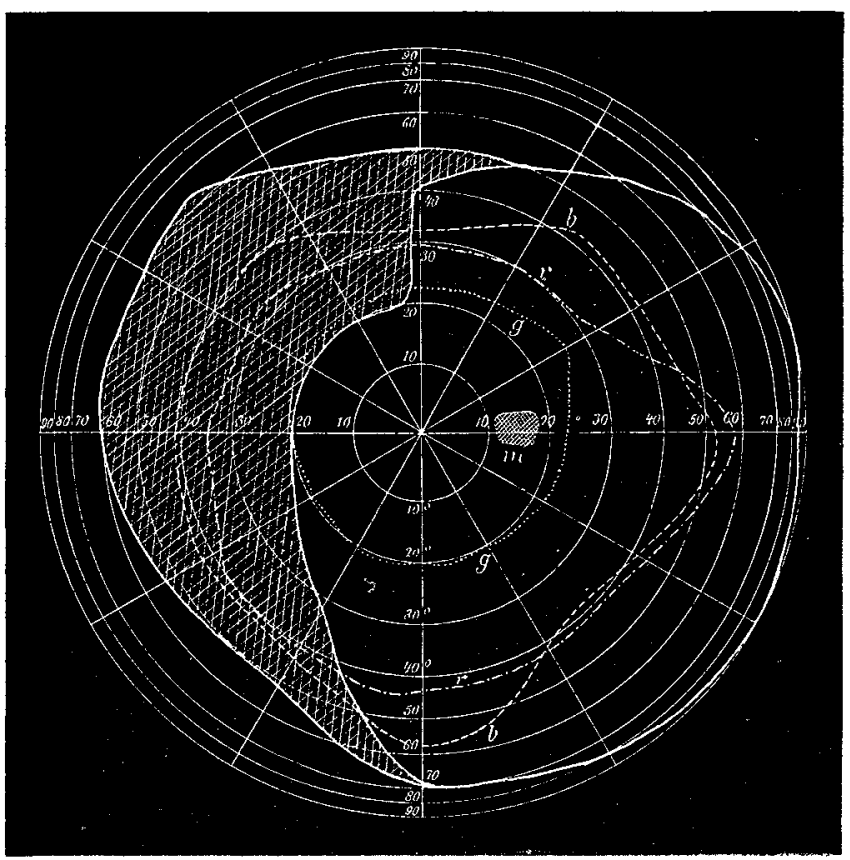

Anatomie 1892, Bd. CXXIX, Heft 2) verwiesen, welche zeigen, wie oft bei pathologischem Verhalten der Schilddrüse beim Menschen auch die Hypophysis krankhafte Veränderungen zeigt

Am schwierigsten wiirde jedenfalls bei einer derartigen Deutung des ganzen Krankheitsprocesses auf einer gemeinsamen Grundlage die doppelseitige Ophthalmoplegia interna bei unserer $\mathrm{Pa}$ tientin zu erklären sein. Zwar ist aus der Litteratur bekannt, dass von den Bewegungsnerven des Auges bei der Hypophysisvergrösserung schon llach seiner anatomischen Lage der Nervus oculomotorius in erster Linie mitbetheiligt wird und dass hierbei auch, wie z. B. bei einzelnen Beobachtungen von Akromegalie mit Hypophysisvergrösserung nachgewiesen ist, Convergenzparese und Ophthalmoplegia interna als isolirte paretische Erscheinungen von seiten der Oculomotorii sich finden können; doch erscheint es mir zu gewagt, bei unserer Patientin diese Erscheinung so erklären zu wollen. Selbst angenommen, eine Vergrösserung der Hypophysis sei wirklich der Grund für die Sehstörung, so ist doch noch nicht einzusehen, wie eine solche Vergrösserung der Hypophysis lediglich das Centrum für Accommodation und Sphincter pupillae beeinträchtigen konnte, ohne die Function der übrigen Oculomotoriusäste zu stören

Bei diesen Schwierigkeiten in der Deutung der Erscheinungen ist vielleicht in zweiter Linie daran zu denken, dass Syphilis die eigentliche Grundlage des ganzen Leidens ist und dass eine gummöse Erkrankung des rechten vorderen Tractusendes mit Uebergreifen auf das Chiasına einerseits die eigenthümliche Sehstörung und Gesichtsfeldbeschränkung hervorbrachte, auf der anderen Seite aber auf Grundlage von Syphilis auch die doppelseitige Ophthalmoplegia interna entstand. Die deutliche Einwirkung der antisyphilitischen Cur spricht für diese Auffassung. Bei der nun schon längere Jahre bestehenden Entartung und Vergrösserung der Schilddrüse, die in letzter Zeit noch zugenommen hat, könnte dann der Ausbruch der Basedow'schen Krankheit durch den Eintritt der intracraniellen Erkrankung begünstigt worden sein.

Die dritte Möglichkeit bei der Deutung, die Basedow'sche Erkrankung als das Primäre und die Sehstörungen als secundär und davon abhängig anzusehen, scheint mir am wenigsten für sich zu haben. Zwar giebt es in der Litteratur einzelne Beobachtungen, wo Morbus Basedowii sich mit Sehnervenatrophie complicirte (Emmert, v. Graefe's Archiv für Ophthalmologie XXII 1, S. 203 und Pedrono, "Des Lésions oculaires dans le goître exophtalmique“ Paris 1885), und ebenso eine v. Story (Ophthalmic rewiew 1883 S. 161), wo doppelseitige Neuritis optica angetroffen wurde, doch sind diese Fälle bei ihrer grossen Seltenheit wohl aus zufälligen Complicationen zu erklären. Eine Combination des Morbus Basedowii mit Tractushemianopsie wie in unserem Falle habe ich in der Litteratur bisher nicht auffinden können und möchte daher bei 
unserer Kranken auch an eine zufällige Complication in erster Linie denken.

Ist es mir somit auch nicht möglich gewesen, eine befriedigende einheitliche Erklärung des ganzen Symptomcomplexes zu geben, so erscheint mir doch das gesammte Krankheitsbild so selten und interessant, dass ich die Vorstellung der Patientin für geboten hielt. 